\title{
NUMERICAL STUDY OF THE EFFECTS OF BOAT-TAIL GEOMETRY ON THE WAKE DYNAMICS OF A BLUFF BODY
}

\author{
U. Caylan ${ }^{1}$, M.I. Yaras ${ }^{1}$, B.R. McAuliffe ${ }^{2}$ \\ ${ }^{1}$ Dept. of Mechanical and Aerospace Engineering, Carleton University, Ottawa, Canada \\ ${ }^{2}$ National Research Council of Canada, Ottawa, Canada
}

\begin{abstract}
This study aims to shed light on the wake dynamics of a bluff body with a streamlined fore-body and a rectangular blunt base through numerical analysis. Specifically, the study was designed to establish the role of instability modes and small-scale turbulence in shaping the wake dynamics, and the effect of a boat-tail including a lobed configuration on the wake structure. In the absence of smallscale turbulence, the near wake of the body is dominated by the effects of two instability modes that promote larger-scale transient motions. Small-scale turbulence is found to have a significant suppressing effect on these motions. Presence of a planar boat-tail is observed not to alter the fundamental structure of the wake, but the relative roles of the instability modes affecting the wake and the resultant streamwise mixing rates are modified. The planar boat-tail is noted to reduce the initial size of the wake significantly to yield substantial reduction in form drag. Shaping the tail into a lobed trailing edge is found to modify the initial cross-section of the wake to an extent to negate the drag-reduction benefits produced by a planar tail. Furthermore, the lobed configuration introduces three-dimensional coherent vortical structures at the tail trailing edge that dominate the near-wake development as well as promoting cross-stream mixing farther downstream.
\end{abstract}

Keywords- bluff-body aerodynamics; boat-tail; lobed mixer; flow instability; wake dynamics

\section{INTRODUCTION}

Bluff bodies are encountered in many practical aerodynamic applications such as bridges, buildings, and ground transportation. Unlike their streamlined counterparts, such bodies experience large regions of flow separation. The presence of separated flow in the wake of bluff bodies results in relatively large magnitudes of form drag. Further, the aerodynamic forces associated with the large-scale transient flow structures prevailing in the wake may be hazardous in certain applications. Active and passive control techniques to counter the adverse effects of the wake region have therefore attracted increasing engineering interest in recent decades. One of the application areas that have been stimulating such research is the transport truck industry with the objectives of improved fuel efficiency, vehicle stability enhancement, wake hazard minimization, and aerodynamic noise reduction. Devices appended to the rear of the trucks and their trailers have been found to be most promising in yielding reduction in aerodynamic drag [1]. One such device, referred to as a boattail, consists of flaps mounted along the perimeter of the trailing end of the trailer, with the flaps oriented to guide the flow towards the center of the wake region [2].

A trailing-edge geometry that has been used with great success for mixing co-flowing streams is the lobed mixer (Fig. 1). Considering the wake region next to the base of a bluff body as a "slow-moving stream", in principle the lobed mixer may be effective in promoting mixing between the core of the wake and its surroundings. The primary mechanism driving mass and momentum exchange between co-flowing streams of fluid is the Kelvin-Helmholtz instability that is promoted inviscidly by the inflectional velocity profile of the shear layer immediately downstream of the lobes. Spanwise-oriented vortices, also known as normal vortices owing to their orientation relative to the primary flow direction, are generated as a result of the roll-up of the free shear layer at the interface of the two streams through this instability mechanism. The streamwise spacing of these normal vortices tends to double with sufficient downstream distance because of a secondary inviscid instability mode that results in vortex pairing, similar to what has been observed in planar free-shear layers [4]. As the normal vortices convect downstream, the relatively larger amount of lower-momentum fluid prevailing in the trough region causes the trough segments of the normal vortices to be progressively stretched in the upstream direction [5]. The shear layer shedding off the trailing edge of the sidewall of the lobe contains vorticity with a streamwise component, and rolls into a streamwise vortex under its self-induced velocity field. The mutual interaction of the streamwise and normal vortices results in their deformation as shown schematically in Fig. 1, with the normal vortices bulging in the trough and crest regions. With streamwise distance, these bulges grow until the normal vortices are broken apart in the regions of pinch-off, triggering the breakdown of the coherent vortical structures into smaller scale turbulence [6].

This study was funded by the National Research Council of Canada. 


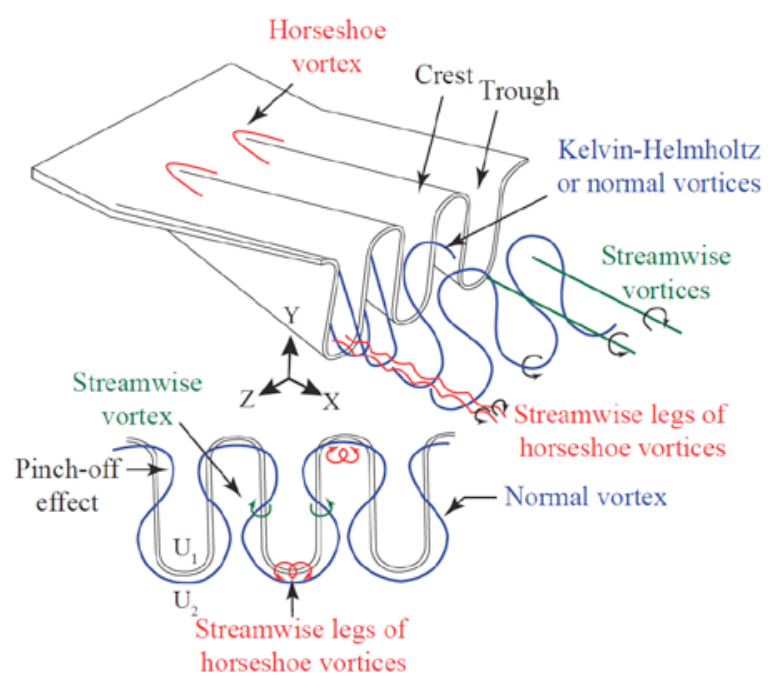

Figure 1 Mixing of co-flowing streams downstream of a lobed trailing edge. (adapted from [3])

Despite extensive research spanning several decades, the dynamics in the wake region of bluff bodies is still not sufficiently well understood. This lack of understanding has hindered further optimization of passive flow control techniques for the wake region, such as the boat-tail. In the context of tractor-trailers, the challenge stems, in part, from the mutually interacting aerodynamic effects of the geometric features of the vehicle spanning a broad range of length scales. A layered approach wherein the effects of individual geometric features on the prevailing aerodynamics are studied first in isolation and then in combinations of increasing complexity has often proven to be an effective strategy. This strategy is adopted in the present study by utilizing a bluff body shaped to produce a well-defined boundary layer at the trailing edge. Combining the lobed-mixer concept with the boat-tail concept may yield a tail geometry with improved performance than the configurations where these devices are utilized separately. Investigating the effectiveness of this lobed boat-tail configuration in comparison to the planar boat-tail configuration constitutes the primary objective of the present study. The study is based on numerical integration of the governing unsteady Navier-Stokes equations in Reynoldsaveraged form.

\section{GEOMETRY OF THE BLUfF BODY AND ITS TAILS}

The geometry of the idealized bluff-body considered in the present study is presented in Fig. 2. The aspect ratio of the trailing-edge cross section $(W / H)$ is set to that of the standard truck trailers in North America. A notably more streamlined upstream portion is chosen for the bluff-body model compared to the shape of a tractor-trailer to ensure dominance of the wake dynamics in the overall aerodynamic drag. The tail illustrated in the figure is studied for both the lobed and planar ( $\varepsilon_{\mathrm{c}}=\varepsilon_{\mathrm{t}}=0$ deg.) configurations.
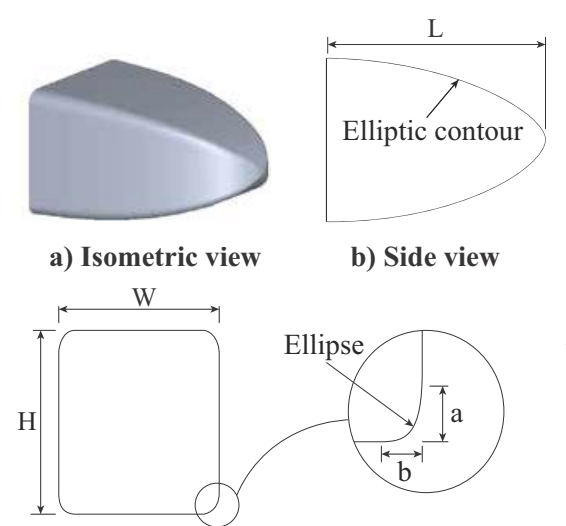

$\mathrm{L} / \mathrm{H}=1.444$

$\mathrm{W} / \mathrm{H}=0.867$

$\mathrm{a} / \mathrm{H}=0.100$

$\mathrm{b} / \mathrm{H}=0.087$

$\mathrm{D}_{\mathrm{h}} / \mathrm{H}=2 \mathrm{~W} /(\mathrm{H}+\mathrm{W})=0.929$

c) Front View
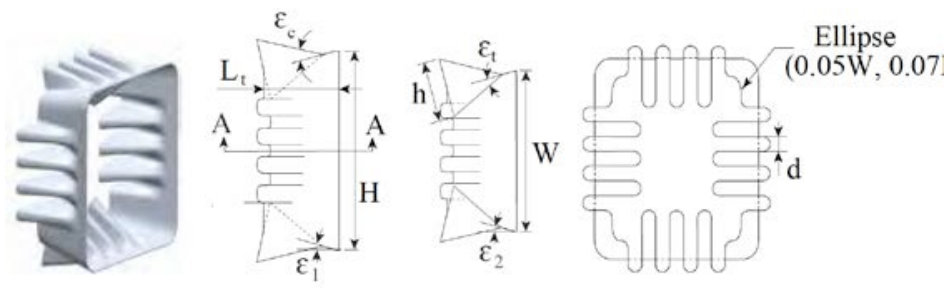

Side view Section A-A

Rear view

$$
\begin{aligned}
& \mathrm{L}_{\mathrm{t}} / \mathrm{H}=0.40 \\
& \mathrm{~h} / \mathrm{H}=0.867 \\
& \mathrm{~d} / \mathrm{H}=0.10 \\
& \varepsilon_{\mathrm{c}}=25.0 \mathrm{deg} . \\
& \varepsilon_{\mathrm{t}}=25.0 \mathrm{deg} . \\
& \varepsilon_{1}=13.0 \mathrm{deg} . \text { (lobed); } 11.0 \mathrm{deg} . \text { (planar) } \\
& \varepsilon_{2}=13.0 \mathrm{deg} .
\end{aligned}
$$

Figure 2 Geometry of the bluff-body and its tails.

\section{COMPUtATIONAL Model AND SOlution Method}

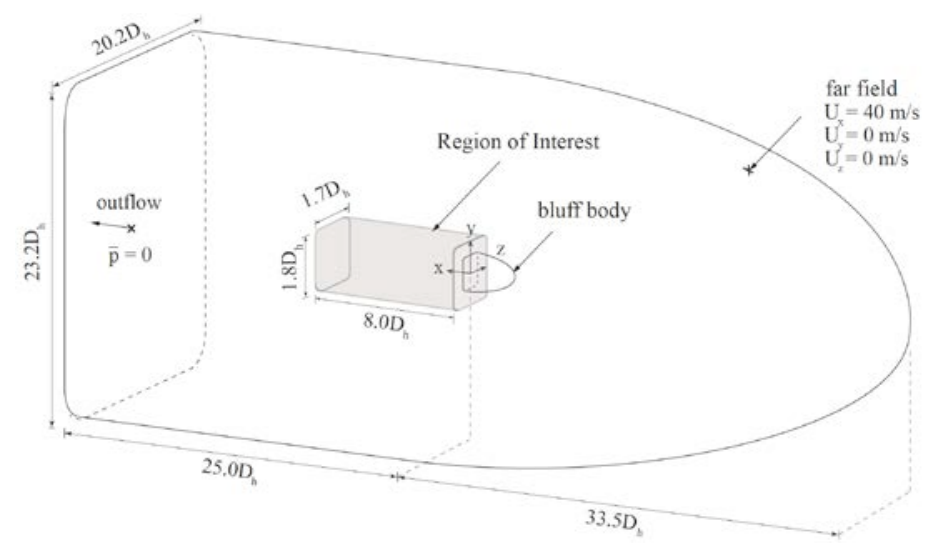

Figure 3 Computational domain.

The computational domain and its boundaries are shown in Fig. 3. The domain is sized notably larger than the region of 
interest to allow for straightforward implementation of boundary conditions on the domain boundaries while avoiding any non-physical influence of these boundary conditions on the flow in the vicinity of the model and its wake. The Region of Interest is sized to conservatively include all possible transient flow structures associated with the near wake. The surfaces of the bluff-body and its tails are set to no-slip walls, and the wall thickness of the tail is set to $1.5 \mathrm{~mm}$, corresponding to $0.8 \%$ of the body's hydraulic diameter, $\mathrm{D}_{\mathrm{h}}$. The placement of the far-field boundary is based on potential flow theory, with the boundary located sufficiently far from the model to yield less than $1 \%$ and $1 \mathrm{deg}$. deviation from the far-field values of the velocity magnitude and direction, respectively. The outflow boundary is placed sufficiently far from the model to allow for natural development of the near wake of the model. The far-field velocity magnitude is set to a value that corresponds to a flow Reynolds number value of $5.3 \times 10^{5}$ based on $D_{h}$. The values of turbulence kinetic energy, $\mathrm{k}$, and specific turbulence dissipation rate, $\omega$, normalized by the far-field velocity and hydraulic diameter of the model, are respectively set to $3.75 \times 10^{-5}$ and 0.663 at the far-field boundary. At the outflow boundary, the static pressure is kept fixed in an area-averaged sense at the far-field value $(0 \mathrm{~Pa}$, gauge).

The spatial grid consists of a total of 23 million cells for the baseline case without a boat-tail, 28 million cells for the planar boat-tail case, and 34 million cells for the lobed boat-tail case. A combination of prism, tetrahedral and hexahedral cells are used to construct the grid, as shown in Fig. 4 for the lobed tail case. The spatial rate of change of grid-cell size is kept below $20 \%$ to avoid excessive truncation errors in the discretization of the spatial derivatives. The cell counts are established through a grid sensitivity analysis, with the computed flow field deemed grid independent when the change in the local velocity vector magnitude and direction anywhere in the domain remains below $1 \%$ upon simultaneous doubling of the grid cell count in each of the three coordinate directions in the wake and vicinity of the bluff body. Along the solid boundaries, the grid cells are clustered using inflation layers containing prism elements to resolve the boundary layer. The wall-normal extent of the flow region covered by the inflation layers is confirmed to include the full thickness of the local boundary layer. The number of inflation layers residing in the boundary layer varies from 9 near the nose of the bluff body to 21 at the trailing edge. Hexahedral cells are used in the Region of Interest and they are clustered within the regions where spatial gradients in velocity are anticipated to be relatively higher. The normalized distance for the first grid node off the wall is set to 0.8 as required for the low-Reynolds-number turbulence model used in the present simulations. The normalization of this distance is based on the viscous length scale, $v / u_{\tau}$, where $v$ is the kinematic viscosity, $u_{\tau}=\left(\tau_{\mathrm{w}} / \rho\right)^{0.5}$ is the friction velocity, $\tau_{\mathrm{w}}$ is the local wall shear stress calculated at the trailing edge of the bluff-body model, and $\rho$ is the fluid density.

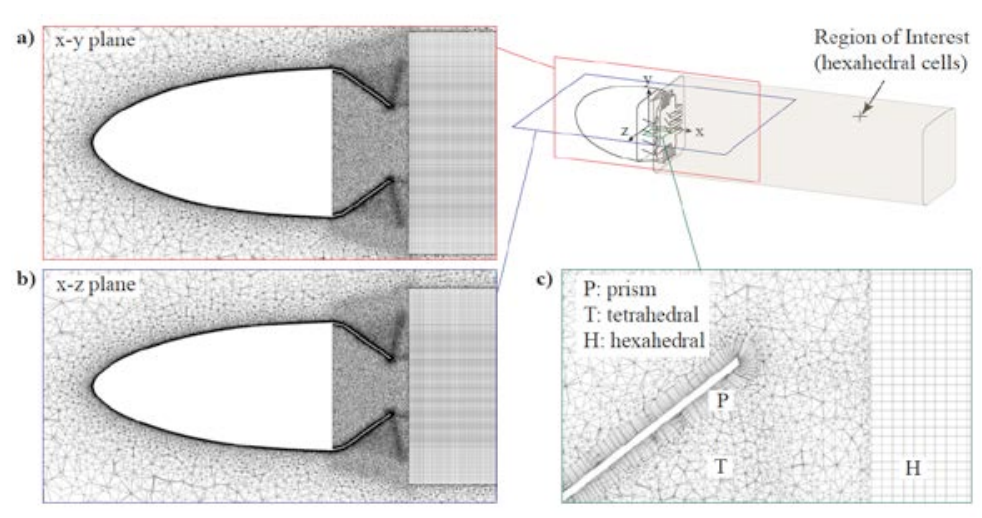

Figure 4 Computational grid.

A time-step size of $2.5 \times 10^{-5} \mathrm{~s}$ is used based on the anticipated transient motion of the large-scale flow structures in the wake of the bluff body model. This time-step size corresponds to a Courant number $(\mathrm{U} \Delta \mathrm{t} / \Delta \mathrm{x})$ value of 0.45 based on the undisturbed far-field velocity and the average cell size of the spatial grid. The solution is marched in time until a statistically steady state is reached in the wake region, and is continued for a sufficient number of time steps to include over 100 cycles of the dominant frequency of shedding from the trailing edge of the body.

A commercial computational fluid dynamics (CFD) software, ANSYS-CFX (version 17), is used to solve the incompressible form of the unsteady Reynolds-averaged Navier-Stokes (RANS) equations with the $\mathrm{k}-\omega$ shear-stress transport (SST) model providing turbulence closure.

\section{RESUlTS AND Discussion}

The wake dynamics is dominated by the vorticity-carrying free-shear layer shedding off the body and the transient vortical structures that develop from this shear layer via a multitude of inviscid instability mechanisms. As such, an effective means of visualizing the near-wake dynamics is through isosurfaces of the second invariant of the velocity gradient tensor, Q, expressed in Cartesian coordinates as:

$Q=\frac{1}{2}\left(\frac{\partial U_{i}}{\partial x_{i}} \frac{\partial U_{j}}{\partial x_{j}}-\frac{\partial U_{i}}{\partial x_{j}} \frac{\partial U_{j}}{\partial x_{i}}\right)$

where $U_{i}$ and $U_{j}$ represent the Cartesian components of the velocity vector, with Einstein summation applied over the indexed terms. The value of $\mathrm{Q}$ at which the isosurfaces are plotted is chosen to most clearly show the vortical structures, which are deemed to potentially have a notable effect on the dynamics of the wake. The isosurfaces are coloured by the normalized streamwise velocity, $\mathrm{U}_{\mathrm{x}} / \mathrm{U}_{\text {ref }}$.

The only dominant feature in the wake region of the baseline configuration (Fig. 5a) is noted to be a steady recirculation zone residing within the first hydraulic diameter streamwise distance of the base. Farther downstream of the wake, there is some evidence of coherent vortical structures, 
a)

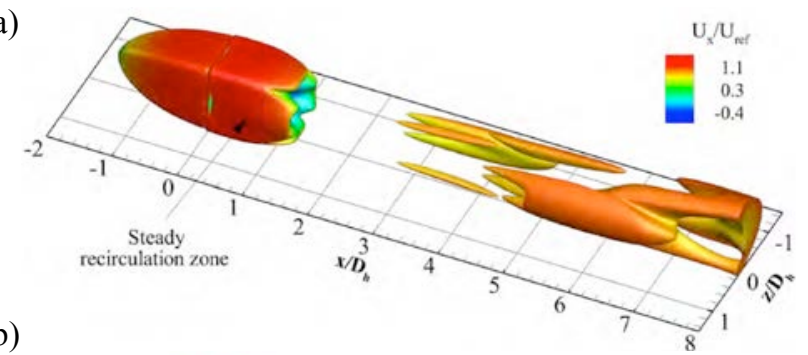

b)

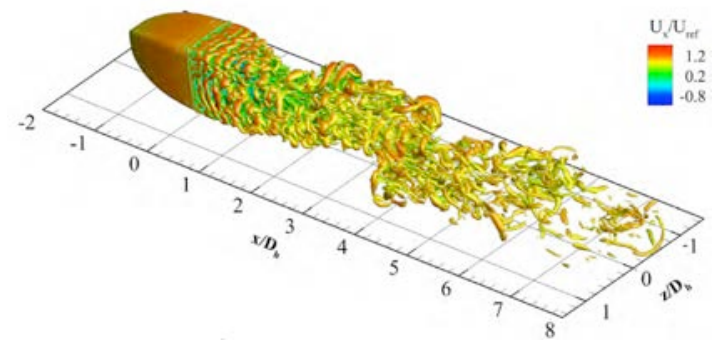

Figure 5 Instantaneous isosurfaces of $\mathrm{Q}$ in the wake region for the baseline configuration a) with nonzero eddy viscosity (isosurface value: $Q /\left(U_{\text {ref }} / D_{\mathrm{h}}\right)^{2}=$ $\left.1.2 \times 10^{-3}\right)$; b) with zero eddy viscosity (isosurface value: $\mathrm{Q} /\left(\mathrm{U}_{\text {ref }} / \mathrm{D}_{\mathrm{h}}\right)^{2}=9.5$ ).

which may plausibly form through amalgamation of smaller vortical structures. To establish the extent of prevailing instability mechanisms in the wake, the effects of which may have been obscured by the mixing effect of small-scale turbulence, a simulation was performed with the Reynolds stresses set to zero magnitude (zero eddy viscosity). The resultant wake is visualized through isosurfaces of Q in Fig. 5b. On the larger scale, the bluff-body wake is note to be dominated by a streamwise-aligned helical pattern that is remarkably similar to the well-studied wake of a sphere [7]. On the basis of this similarity, it may be argued that this helical pattern is the result of the same mechanism as that observed for spheres, namely a local absolute instability which develops just upstream of the rear stagnation point of the recirculation zone located at $x / D_{h}=1.4$. The pitch of the helical pattern in Fig. $5 b$ corresponds to an orbit speed of the local absolute instability that is equal to $1.4 \mathrm{U}_{\text {ref }} / \mathrm{D}_{\mathrm{h}}$. This angular speed is noted to be consistent with the observations in the wake of spheres [7]. Even though the sense of rotation of the helical pattern remained the same throughout the temporal length of the present simulation, it is quite plausible that this could alternate between clockwise and counter-clockwise directions randomly over longer time periods, similar to what has been observed in the wake of axisymmetric bluff bodies $[7,8]$. In close vicinity of the base for the zero eddy viscosity case (Fig. 5b), the boundary layer separating along the base perimeter is observed to form a train of vortex rings, which form due to the wellknown Kelvin-Helmholtz inviscid instability mode triggered by the inflectional velocity profile of the shear layer immediately downstream of the location of separation. The streamwise spacing of the vortex rings yields a vortex shedding frequency that corresponds to a Strouhal number of 6.0 based on the hydraulic diameter, $D_{h}$. This Strouhal number value is in favourable agreement with published results in the near wake of bluff bodies [7]. The absence of the noted instability-driven flow patterns when the local eddy viscosity values are evaluated via the turbulence model (Fig. 5a) illustrates the dominant role of small-scale turbulence in the overall wake development for this baseline configuration.

a)

b)
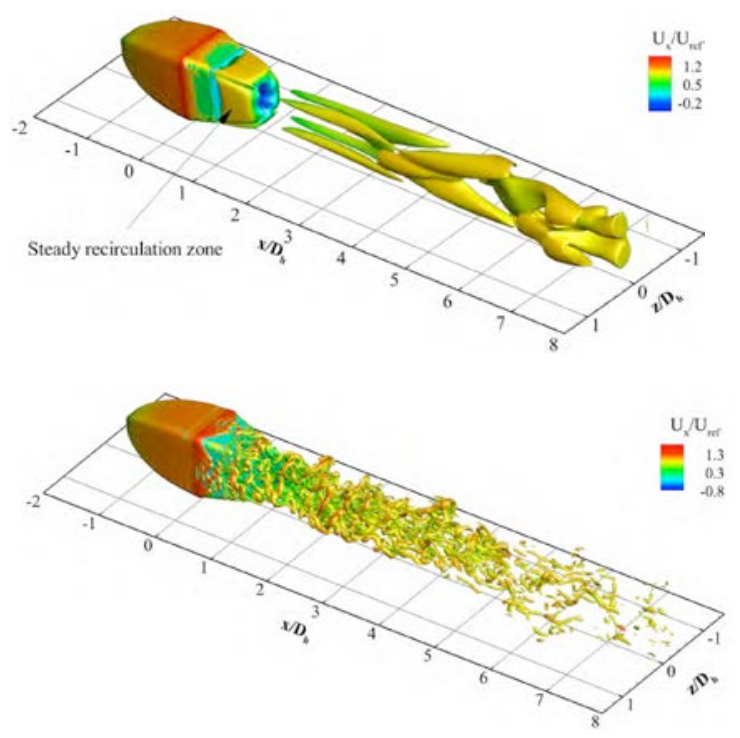

Figure 6 Instantaneous isosurfaces of $\mathrm{Q}$ in the wake region for the planar boat-tail configuration a) with nonzero eddy viscosity (isosurface value: $\left.\mathrm{Q} /\left(\mathrm{U}_{\text {ref }} / \mathrm{D}_{\mathrm{h}}\right)^{2}=2.4 \times 10^{-2}\right)$; b) with zero eddy viscosity (isosurface value: $\left.\mathrm{Q} /\left(\mathrm{U}_{\text {ref }} / \mathrm{D}_{\mathrm{h}}\right)^{2}=9.5\right)$.

The effect of a boat-tail on the wake development is illustrated through isosurfaces of $\mathrm{Q}$ in Fig. 6. Comparing the structure of this flow field in the absence of the mixing effects of small-scale turbulence (Fig. 6b) to that of the baseline configuration (Fig. 5b), one notes a notable weakening of the helical pattern. This suggests that the local rotational instability in the recirculation zone responsible for the helical wake shape may either be absent or be substantially reduced in strength in the presence of the tails. The boundary layer is separated on the tail surfaces, which yields a highly inflectional surface-normal profile of streamwise velocity, which is inviscid unstable. This instability results in streamwise grouping of spanwise vorticity in the separated shear layer. This fundamental instability process has been thoroughly studied in the context of separation bubbles on airfoils [9]. Following this streamwise grouping, yet another instability promotes waviness of the spanwise vorticity lines, which triggers a self-induction process to form hairpin-like vortices as observed in Fig. 6b. This development appears suppressed at the four rounded corners of the boat-tail, plausibly due to differences in the characteristics of the boundary layer at the leading end of the tail at these corners compared to the flat sections of the perimeter. The wake cross section with the boat-tail is reduced compared to the baseline case, which is well-known to be the primary effect of boattails to realize higher base pressures hence reduced drag on bluff bodies. The effect of small-scale turbulence on the wake flow field observed by contrasting Fig. 6b with Fig. 6a is somewhat similar to what was noted for the baseline 
configuration in Fig. 5. The boundary layer separation on the tail surfaces is not as severe as for the zero- eddy-viscosity case, hence the aforementioned instability-driven streamwiseperiodic flow development is suppressed. The near wake develops into a steady recirculation zone, and weak largerscale vortical structures prevail farther downstream. The vortical structures appear in pairs, which may suggest that they exist as hairpin-like vortices only parts of which are visible in this plotting format. The streamwise spacing of these pairs appears to be periodic, corresponding to a Strouhal number of 0.7 . This streamwise periodic formation of coherent vortical structures is similar to the wakes of spheres.

Shaping the trailing edge of the tail into lobes modifies the wake dynamics significantly as illustrated through isosurfaces of $\mathrm{Q}$ in Fig. 7. As was noted in the Introduction, lobed mixers promote mixing by increasing the contact surface area between the streams to be mixed and by developing additional coherent vortical structures. The increased surface area due to the lobes is observed to increase the cross sectional area of the wake significantly compared to that of the planar boat-tail configuration (Fig. 7b), to the extent that the cross section of the wake is now comparable to that of the no-tail baseline configuration (Fig. 5b). Accordingly, in the context of the manner a straight tail increases the base pressure, the presence of the lobes with the specific lobe geometries considered may offset the drag benefits of a tailed configuration.

The development of the so-called normal vortices, their streamwise tilting, and their deformation including the pinchoff effect of streamwise vortices shedding of the side walls of the lobes, all of which are well-established dynamics as reviewed in the Introduction, produces a well-defined spanwise-periodic vorticity pattern immediately downstream of

a)
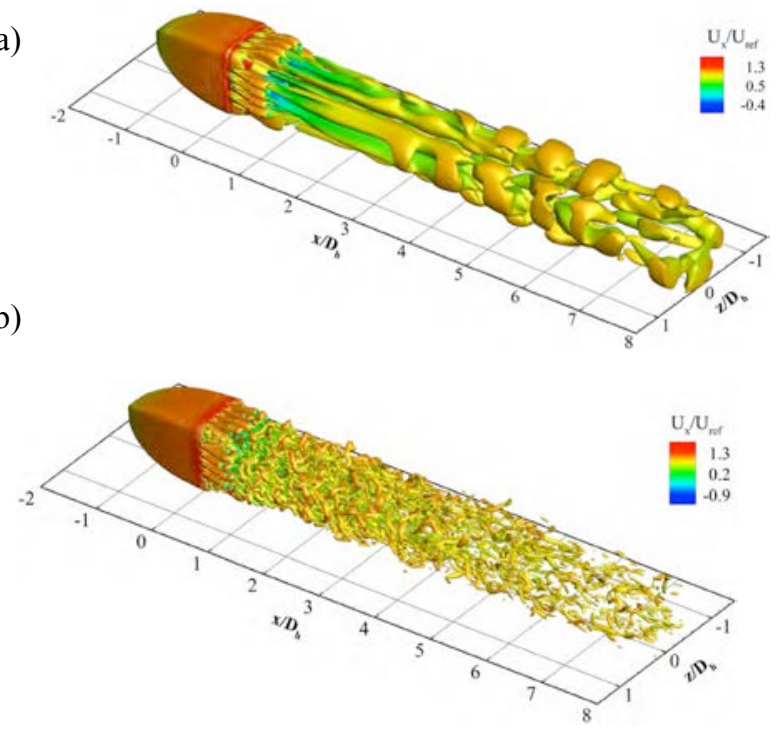

Figure 7 Instantaneous isosurfaces of Q in the wake region for the lobed boattail configuration a) with nonzero eddy viscosity (isosurface value: $Q /\left(U_{\text {ref }} / D_{h}\right)^{2}$ $\left.=2.4 \times 10^{-2}\right)$; b) with zero eddy viscosity (isosurface value: $Q /\left(U_{\text {ref }} / D_{h}\right)^{2}=9.5$ ).

the lobes as illustrated in Figure 7b. The contribution of these vortical structures to the wake dynamics becomes notably more evident when one compares the wake of the lobed tail and planar tail configurations for nonzero-eddy-viscosity conditions (Figs. 6a and 7a). The streamwise- periodic coherent vortical structures that occur at a Strouhal number of 0.7 for the planar tail configuration are replaced by streamwise-periodic vortex loops that occur more frequently corresponding to a Strouhal number of 1.0.

Based on the wake dynamics discussed in the foregoing, one would expect the body drag force for the planar boat-tail configuration to be lower than for the baseline configuration, whereas the lobed boat-tail configuration to yield a drag force comparable to that of the baseline configuration. These expected trends are confirmed by the value of the drag coefficient on the body for the baseline, planar boat-tail and lobed boat-tail configurations, which were calculated by integration of the surface pressures to be $0.22,0.09$ and 0.25 , respectively.

Experiments were conducted in a wind tunnel with bluffbody and tail geometries that match those considered in the computational study at dynamically similar operating conditions. The hydraulic diameter of the wind-tunnel bluffbody model was $195 \mathrm{~mm}$. The model was tested in a closedcircuit wind tunnel with a test section cross-sectional area of $508 \mathrm{~mm} \times 762 \mathrm{~mm}$. Slotted walls were used on the test section to minimize the effect of wall interference, and the drag force on the model was measured with a load balance. The drag coefficient values based on the measured drag force was 0.26 , 0.12 and 0.27 for the baseline, planar tail and lobed tail configurations, respectively. The favourable comparison of the trends in the computational and experimental drag coefficients among the three configurations supports confidence in the computed results.

\section{CONCLUSIONS}

The present study investigated the wake dynamics of a bluff body with a streamlined fore body and a rectangular blunt base of 1.15 aspect ratio. In the absence of small-scale turbulence, the near-wake development is shown to resemble that of a sphere rather that of box-shaped bluff bodies of comparable base aspect ratios. Specifically, the wake is observed to develop a helical pattern approximately aligned with the streamwise direction, which is the result of a local rotating instability within the recirculation region by the base of the body. Another dominant feature of the wake is the formation of vorticity rings immediately downstream of the edge of the base, prompted by the Kelvin-Helmholtz instability of the free shear layer that forms as an extension of the boundary layers of the body. The wavelength of the helical pattern and the formation frequency of the vortex rings are found to agree well with those prevailing in the wake of spheres. The noted wake structure is shown to persist at flow Reynolds numbers as high as 512,000 provided the small-scale turbulence in the flow is suppressed. In the presence of such turbulence, the helical pattern as well as periodic shedding of vorticity rings are found to be suppressed. 
In the presence of a planar tail, the helical wake pattern observed for the baseline configuration is weakened significantly. Boundary-layer separation on the tail surfaces is observed to produce periodic streamwise grouping of spanwise vorticity through an inviscid instability, which is followed by formation of hairpin-like vortical structures through a secondary instability. The initial wake cross section is notably reduced in the presence of the tail, as the tail promotes movement of freestream fluid into the wake region. As with the baseline configuration, small-scale turbulence is observed to significantly suppress the dynamics driven by multiple instability modes in the laminar scenario. In the presence of small-scale turbulence, the wake is dominated by a steady recirculation zone in close vicinity of the base, and by streamwise-periodic vortex loops farther downstream.

In the presence of lobes on the tail, the wake dynamics are observed to differ significantly from those of the planar-tail configuration. Specifically, the initial wake cross-section is notably larger, with a size that is comparable to that of the baseline configuration. The well-known coherent vortical structures associated with lobed mixers are observed to dominate the wake in close proximity of the trailing edge of the tail. In the absence of mixing via small-scale turbulence, there is no evidence of a helical pattern in the wake unlike the other two cases, which suggests that the rotational instability responsible for this pattern may no longer develop in the presence of the vortical structures created by the lobed trailing edge. The mixing enhancement effect of these vortical structures persists for a notable streamwise distance, including in the presence of small-scale turbulence. Despite this enhanced mixing, the drag-reduction benefits of a straight tail are lost when lobes are added to the tail. This is likely caused by the lobes diverting the freestream away from the core of the wake while they develop vortical structures that promote crosssteam mixing.

\section{ACKNOWLEDGMENT}

Financial support for this study provided by the National Research Council of Canada is gratefully acknowledged.

\section{REFERENCES}

[1] N. Schaut and R. Sengupta, "Aerodynamic optimization of trailer add-on devices fully- and partially-skirted trailer configurations," SAE Inter. J. Commer. Vehicles, 8, pp. 695-704, 2015.

[2] B.R. McAuliffe and A. Wall, "Aerodynamic performance of flat-panel boat-tails and their interactive benefits with side-skirts," SAE Int. J. of Comm. Vehicles 9(2), pp. 70-82, 2016.

[3] D.C. McCormick and J.C. Bennett, "Vortical and turbulent structure of a lobed mixer free shear layer," AIAA Journal, 32, pp. 18521859, 1994.

[4] C.D. Winant and F.K. Browand, "Vortex pairing: the mechanism of turbulent mixing-layer growth at moderate Reynolds number," J. Fluid Mech., 63, pp. 237-255, 1974.

[5] J. Brinkerho $\square$, H. Oria and M.I. Yaras, (2013). "Experimental and computational study of mixing mechanisms in an axisymmetric lobed mixer," J. Propulsion and Power, 29(5), pp. 1017-1030, 2013.

[6] L. Ukeiley, M. Varghese, M. Glauser and D. Valentine, "Multifractal analysis of a lobed mixer flow field utilizing the Proper Orthogonal Decomposition," AIAA Journal, 30, pp. 1260-1267, 1992.

[7] E. Berger, D. Scholz, and M. Schumm, "Coherent vortex structures in the wake of a sphere and a circular disk at rest and under forced vibrations," J. Fluids and Structures, 4(3), pp. 231-257, 1989.

[8] S. Taneda, "Visual observations of the flow past a sphere at Reynolds numbers between 104 and 106," J. Fluid Mech., 85, pp. 187-192, 1978.

[9] B.R. McAuliffe and M.I. Yaras, "Numerical study of instability mechanisms leading to transition in separation bubbles," J. Turbomachinery, 130(2), 021006, 2008. 